\title{
Códigos corporales y tecnológicos: Los feminismos como prácticas hacker *
}

\author{
Lola Martínez Pozo**
}

\begin{abstract}
Resumen
En el presente artículo destaco las principales críticas feministas a la tecnociencia $y$ las contribuciones procedentes de las heterogéneas aproximaciones feministas a las tecnologías. El objetivo se dirige a desarrollar una lectura que sitúa en un mismo plano cuerpo y tecnología para interpretar los feminismos como prácticas hacker a sistemas normativos. Dicha lectura es derivada de las reflexiones propiciadas por la experiencia etnográfica con colectivos, trayectorias y proyectos que abordan la conexión entre cuerpos, sexualidades y tecnologías desde perspectivas feministas y transfeministas.
\end{abstract}

Palabras clave: Cuerpos; Tecnologías; Feminismos; Hacking.

* Recibido el 29 de setembro de 2018, aceptado 14 de agosto de 2019. Agradecimientos a Carmen Gregorio Gil, por sus revisiones y apoyo en todo el proceso de investigación, a Lucía, Klau, Urko, Diego, Ce, Kina y Alex por compartirme sus tiempos, perspectivas, experiencias y proyectos.

** Personal docente e investigadora del Departamento de Antropología Social y adscrita al Instituto Universitario de Estudios de las Mujeres y de Género, y al grupo de investigación "Otras. Perspectivas feministas en Antropología Social", Universidad de Granada, Granada España. lolamartinezpozo@ugr.es / http://orcid.org/0000-0001-7897-2676 
Corporal and Technological Codes: Feminisms as Hacker Practices

\begin{abstract}
In this article, the primary feminist critiques of techno-science and the contribution originating from heterogeneous feminist approaches to technologies are pointed out. The objective directs to develop an interpretation which places body and technology at the same plane in order to read feminisms as hacker practices to normative systems. This reading derives from reflections promoted by the ethnographical experience with groups, career paths and projects that deal with the connection between bodies, sexualities and technologies from feminist and trans-feminist perspectives.
\end{abstract}

Keywords: Bodies, Technologies, Feminisms, Hacking. 


\section{Introducción}

A modo de contextualización, inicio el presente artículo destacando las principales críticas feministas de la tecnociencia como marco epistemológico, metodológico y político de referencia que impulsa el desarrollo de investigaciones y teorizaciones feministas de las tecnologías en la década de los setenta.

A continuación, mediante un recorrido genealógico, expongo un análisis de las diferentes perspectivas feministas sobre las tecnologías, subrayando sus principales contribuciones, divergencias, continuidades y transformaciones.

A partir de dicha genealogía, identifico una mutación radical en las posiciones y prácticas feministas de las tecnologías que permite exceder enfoques centrados en el género, el paradigma de la exclusión y las políticas de acceso para desarrollar análisis más complejos de la tecnocultura y prácticas de agenciamiento, autonomía y apropiación.

En este sentido, establezco una conexión entre cuerpos y tecnologías. A lo largo de estas líneas indago en las corporalidades, subjetividades y tecnologías como superficies codificadas por sistemas operativos tecnoculturales normativos, así como en las heterogéneas articulaciones entre feminismos $y$ hacking. Dicha conexión y articulación las realizo a partir del trabajo de campo etnográfico desarrollado en la ciudad de Barcelona en el contexto activista transfeminista.

Feminismos y tecnociencia: de la tecnofobia a la reapropiación tecnológica

Las heterogéneas corrientes feministas de la tecnociencia emergen en la década de los setenta, orientadas a denunciar el sexismo y androcentrismo predominante en los ámbitos científicotécnicos, así como la escasez, invisibilización, sub-representación y exclusión de las mujeres (Grint \& Gill, 1995; Haraway, 1995[1991], 1997; Harding, 1996[1986]; Keller, 1991; Rose, 1994; Schiebinger, 1993). 
Los iniciales estudios sobre género, ciencia y tecnología se centraron en analizar la ausencia de las mujeres, recuperar sus presencias, así como en proponer estrategias educativas $e$ institucionales dirigidas a potenciar el acceso de las mujeres a los escenarios tecnológicos y científicos. Por lo que los debates feministas sobre ciencia y tecnología tienen su origen en la constatación de la escasez de mujeres y transcienden hasta cuestiones políticas y epistemológicas (González y Pérez, 2002).

Las críticas feministas de la tecnociencia cuentan con cinco décadas de trayectoria poniendo de relieve la articulación mutuamente constituyente entre ciencia, tecnología y género, es decir, identificando cómo los ámbitos científico-tecnológicos se construyen a partir de ideologías y desigualdades de género, a la par que evidenciando cómo estos refuerzan los estereotipos, roles, relaciones y prácticas de género (González y Fernández, 2016).

A pesar de las diferencias entre las diversas perspectivas feministas de la tecnociencia éstas coinciden en enfatizar la coproducción entre género y tecnología. Así mismo, las teorías feministas destacan cómo los cambios tecnológicos influyen y generan nuevas relaciones de poder con una incidencia diferencial de género (Wajcman, 1991), y la necesidad de políticas feministas de la tecnología para lograr relaciones de género equitativas (Wajcman, 2009).

Los estudios feministas sobre tecnología y género tienen como marco de referencia las críticas feministas a la ciencia y el conocimiento científico como ámbitos inherentemente patriarcales (Wajcman, 1991). En este sentido, tomando como referencia los trabajos de autoras como Code (1991), Haraway (1988, 1995[1991]) y Harding (1991, 1996[1986]), las epistemologías feministas aplicadas a diversos ámbitos científicos también han abordado la tecnología, cuestionando el androcentrismo, universalismo y racionalismo mediante el reconocimiento de la relevancia del contexto y corporalidad del sujeto cognoscente, la parcialidad del conocimiento y su vinculación con el poder.

Estos cuestionamientos feministas han incidido críticamente en los ámbitos científico-tecnológicos al igual que los feminismos 
se han ido transformando por los cambios tecnológicos, como muestro más adelante. De tal forma que, en el marco de los feminismos encontramos desde perspectivas más pesimistas, incluso tecnofóbicas, hasta posiciones que apuestan por la reconfiguración y reapropiación tecnológica.

Las corrientes críticas a las tecnologías se iniciaron en el contexto estadounidense en la década de los setenta y, en escasos años, fueron contando con un marcado carácter transnacional (Vergés 2013).

Así mismo, los análisis sobre las tecnologías atraviesan las diferentes corrientes feministas, especialmente, el feminismo liberal, radical-cultural, socialista, poscolonialista, los ciberfeminismos y los feminismos queer, abordando la diversidad de tecnologías (Vergés, 2013).

A partir de la década de los noventa, las teorizaciones feministas de las tecnologías adquieren una especial relevancia y consolidación (Wajcman, 2004), potenciándose el interés por este ámbito desde perspectivas ciberfeministas, tecnofeministas, ciberqueer y tecnoqueer $\mathrm{y}$, más recientemente, desde perspectivas hackfeministas y transfeministas.

Las emergentes críticas feministas de las tecnologías de corte liberal se centraron en el acceso de las mujeres a los escenarios científico-tecnológicos, considerando la masculinización de dichos ámbitos, pero asumiendo el carácter neutral de las tecnologías con respecto al género (Perdomo, 2016; Wajcman, 2009). Estas pioneras investigaciones indagaban en la educación y socialización diferencial de género, en las barreras estructurales de acceso y participación de las mujeres, analizaban la distribución desigual y proponían el desarrollo de medidas educativas y políticas (Vergés, 2013).

De manera simultánea, la crítica al sexismo y androcentrismo presentes en la tecnología y la generización de la misma, es decir, la inclusión de la perspectiva de género en el análisis científico-tecnológico vendría a ser elaborada por corrientes feministas radicales-culturales, socialistas, así como por ecofeministas (Merchand, 1980; Mies y Shiva, 1993). Perspectivas 
que, posteriormente, serían cuestionadas dado su carácter esencialista, victimista y tecnofóbico (Wajcam, 2009).

Concretando en las aproximaciones feministas radicalesculturales, las tecnologías eran consideradas substancialmente patriarcales. Por ello, centraron sus investigaciones en la explotación y dominación de los cuerpos y sexualidades de las mujeres a través de las tecnologías reproductivas, estéticas y la genética (Vergés, 2013).

Por su parte, las críticas feministas socialistas (Cockburn, 1983) abordaron la tecnología como ámbito de dominación masculina forjado mediante la división sexual del trabajo y la exclusión de las mujeres a través de la relación entre masculinidad, cualificación y tecnologías.

Las investigaciones feministas socialistas desarrollaron análisis que vinculaban género y clase para abordar los efectos de los cambios tecnológicos en la vida y empleo de las mujeres en relación con las ocupaciones tecnológicas feminizadas, las condiciones laborales y de explotación (Vergés, 2013).

Así mismo, desde los feminismos socialistas se realizaron estudios sobre la relación de las mujeres, configuradas como usuarias, con las tecnologías consideradas domésticas (Oakley, 1974; Schwartz, 1983).

Por otro lado, a finales de los años ochenta, las anteriores corrientes comenzaron a ser transformadas por las críticas procedentes de los feminismos queer, poscoloniales $y$ posmodernistas, las cuales desarrollaron rupturas epistemológicas que permitieron superar la centralidad del género y la igualdad en los debates y análisis tecnológicos, e incluir la conexión con otros ejes de poder. Así mismo, desde la década de los noventa, a partir de la era digital y el desarrollo de los posfeminismos, se produce un cambio de paradigma radical en las aproximaciones feministas hacia las tecnologías abordando el potencial transformador de las mismas (Wajcman, 2009).

Las diversas posiciones posfeministas de las tecnologías encuentran en el trabajo de Haraway (1995[1991]) la inspiración y el referente para el desarrollo de nuevas posibilidades de análisis y 
prácticas políticas que destacan las potencialidades de agenciamiento crítico y reapropiación tecnológica para la deconstrucción de las categorías sociales y la transformación radical de la tecnociencia.

En este contexto, la aparición de los ciberfeminismos está vinculada con la incursión de las tecnologías de la información y la comunicación y con el consecuente surgimiento de movimientos políticos y artísticos feministas en la red. Los emergentes ciberfeminismos fueron propiciados por VNS Matrix, el movimiento Net-art, la I Internacional Ciberfeminista de Documenta X, Old Boys Network, y la publicación de Ceros+Unos de Sadie Plant (1997). Actualmente, los ciberfeminismos incorporan las diversas experiencias de luchas feministas en la red (Boix, 2015; De Miguel y Boix, 2013), por lo que son un conglomerado plural de teorías, narrativas y prácticas que, mediante la reapropiación de las tecnologías digitales, se orientan a la subversión de la tecnocultura contemporánea en el ámbito de la cultura-red.

En la actualidad, los ciberfeminismos pueden ser considerados como un conjunto ampliamente heterogéneo y disperso de nodos autónomos e interconectados (Boix, 2015), difícil de abordar y unificar. No obstante, siguiendo a Boix (2015) y Vergés (2013) podemos esbozar dos olas ciberfeministas.

En un primer momento, las teorizaciones y prácticas ciberfeministas tenían un marcado carácter tecnofílico y esencialista, compartían una comprensión neutral de las tecnologías y establecían una relación estrecha entre las características de las mujeres y los rasgos de las tecnologías de la información y la comunicación. Estas perspectivas se dirigían al desarrollo de prácticas feministas descorporeizadas-posidentitarias en la red, orientadas a la deconstrucción del género (Plant, 1997).

Por su parte, en una segunda ola, los ciberfeminismos se enfocaron en superar el determinismo tecnológico y el esencialismo sexual, a la par, que continuaban desarrollando políticas feministas en los escenarios digitales orientadas al cuestionamiento y subversión de las identidades, roles y relaciones 
de género (Fernández; Wilding y Wright, 2002). Así mismo, se plantea la utilización de las tecnologías por parte de las mujeres para transcender los imaginarios masculinizados y los valores capitalistas de explotación, dominación y violencia (Braidotti, 1996). En este sentido, desde las teorías y prácticas ciberfeministas se potencia la transformación tanto del género como de la propia tecnología (Vergés, Hache y Cruells, 2010). Igualmente, las prácticas artísticas y activistas feministas habitan la cultura-red generando otras formas de colectividad-conectividad dirigidas a deconstruir la reproducción de las identidades socioculturales normativas, así como a desarticular la división entre público-privado y producción-reproducción (Zafra, 2011).

Por lo que se refiere al tecnofeminismo, concepto elaborado inicialmente por Judtih Wajcman (2004), éste emerge de la vinculación entre los estudios socio-constructivistas y los feminismos en oposición a las tendencias pesimistas feministas de la década de los ochenta y a las posturas tecnofílicas de la primera ola ciberfeminista (Gill, 2005). Los tecnofeminismos proponen la transformación crítica feminista de las tecnologías desde posturas que rechazan el carácter neutral de las tecnologías y enfatizan la persistencia de tradicionales jerarquizaciones de género y la aparición de nuevas relaciones de poder (Wajcman, 2004).

Así mismo, desde perspectivas tecnofeministas la relación de co-producción entre género-tecnología se interpreta prestando atención a las identidades socio-políticas producidas socialmente y políticas producidas y reproducidas en los ámbitos tecnológicos y digitales, y al modo mediante el que se reproducen o subvierten sistemas sociales hegemónicos (Wajcman, 2007).

Por su parte, las perspectivas feministas queer de las tecnologías han permitido exceder la consideración generizada de las mismas, contemplar la sexualidad y la tecnología como mutuamente constituyentes, abordar la identidad y subjetividad en términos no binarios en relación con lo tecnológico, así como cuestionar la heteronormatividad presente tanto en los escenarios tecnológicos como en las corrientes feministas (Lansdtröm, 2007). 
Los feminismos queer de las tecnologías se han desarrollado en diversos ámbitos donde destaco: la visibilización, uso y desarrollo de tecnologías digitales y biotecnologías por parte de comunidades y subjetividades no heterosexuales; la identidad y auto-representación on-line; la creación y participación en redes, espacios y comunidades virtuales; así como la trasformación de prácticas erótico-sexuales a través de las tecnologías (Alexander, 2002; Bryson, 2004; Carlson, 2001; Heinz et alii, 2002; Nip, 2004; O'riordan \& Philips, 2007; Stone, 1991, 1995; Wakeford, 2000, 2002; Woodland, 2000; Wolmark, 1999).

Por último, las críticas procedentes de los feminismos poscoloniales han supuesto una notable contribución en un dominio predominante anglosajón (Vergés, 2013). Desde dichas perspectivas se enfatiza en la interseccionalidad de categorizaciones y ejes de poder, como son el género, la sexualidad, la clase, la raza, el sexismo, la heteronormatividad, el colonialismo y el capitalismo, para abordar el acceso, participación, explotación y desigualdades en los ámbitos tecnocientíficos. De esta forma, inciden en las diferencias geopolíticas entre mujeres desde perspectivas interseccionales, así como en la articulación entre género, raza y tecnología (Daniels, 2009a; Galpin, 2002; Leggon, 2006; Nakamura, 2002; Philip, 2012; Wrigth, 2005). Desvelando, de este modo, el racismo predominante en los escenarios tecnocientíficos y el etnocentrismo característico de las teorizaciones feministas occidentales sobre las tecnologías (Daniels, 2009b; Fernández, 2003; Gajjala, 1999; Kvasny, 2006; Tapia, 2015; Wright, 2002).

En síntesis, las heterogéneas perspectivas feministas a las que me he referido difieren en función de las diversas concepciones de género y tecnología (Wajman, 2007). Si bien, a mi modo de ver, la predominante interpretación de la relación género-tecnología desde categorías binarias ha reducido numerosas indagaciones y prácticas a la cuestión del acceso como única dimensión relevante. En consecuencia, el objetivo de conseguir la igualdad en los ámbitos tecnológicos mediante la eliminación de la brecha digital de género es predominante, pero 
implica reproducir y participar de un modo de producciónconsumo posfordista donde la tecnocultura es organizada por un sistema capitalizado inherentemente desigual (Pujol y Montenegro, 2015).

Por ello, considero necesario desarrollar análisis desde enfoques articuladores de diferencias que aborden las tecnologías como entramados de poder $e$ indaguen en las múltiples dimensiones que implican, con el objetivo de promover perspectivas dirigidas a la subversión del orden tecno social dominante.

\section{Códigos normativos: tecnologías corporales}

De Lauretis (1989) habilitó la noción de tecnología para repensar el género, y con ello el cuerpo. Por su parte, Haraway (1995[1991]) nos dotó de la imaginería cyborg como herramienta analítico-política que nos permite identificar y subvertir las contemporáneas tecnologías del cuerpo. Así mismo, Preciado (2008) dio cuenta de la relevancia de la tecnociencia en la programación de nuestros cuerpos en el régimen farmacopornográfico. Donde la producción de subjetividades opera a través de tecnologías que se incorporan y penetran en los cuerpos.

En esta línea, desarrollo una lectura que sitúa en un mismo plano cuerpo y tecnología; programación, codificación y corporeización; así como códigos tecnológicos y códigos corporales. Tales articulaciones cuentan con la potencialidad política de generar nuevos espacios, subjetividades, narrativas y prácticas que resistan y transgredan sistemas tecnoculturales normativos.

En este sentido, vincular cuerpo y tecnología nos permite desarrollar una fructífera articulación al interpretar los códigos reguladores de la tecnología, que tienen como resultado los productos y servicios privativos y corporativos, en conexión con los códigos culturales corporales, que establecen rígidos modelos identitarios binarios y jerárquicos construidos en torno al sexo, el 
género, la sexualidad, la raza, la capacidad y múltiples diferenciaciones sociales.

De manera habitual, las narrativas sobre la tecnología localizan al cuerpo en planos diferenciados del código y lo tecnológico. En oposición, en trabajos como el de Douglas Thomas (2005) se demuestra cómo la tecnología incorpora aspectos como código, cuerpo y performance.

La comprensión de la vinculación entre cuerpo y performance nos puede resultar más fácilmente asumible que la conexión entre cuerpo y código, debido a que este último tiende a entenderse en términos de abstracción matemática e informática (Thomas, 2005).

El código es el núcleo de la tecnología, es un sistema de regulación normativo estructurado y estructurante (Thomas, 2005). La función normativa del código se desarrolla en términos abstractos, estableciendo los límites de la normalidad (Lessig, 1999). Así mismo, el código se basa en la repetición continuada sin alterabilidad ni diferencia. Al código le subyace una estructura y proceso de escritura que desaparece cuando este produce su efecto final (Thomas, 2005). El código, es el elemento regulador que permite (re)definir, constantemente, por parte de sus creadorxs, diversos escenarios y aparatos tecnológicos, estableciendo las posibilidades y los límites. Por ello, la privatización del código implica su comercialización por parte de corporaciones tecnológicas y el incremento del control desde entidades gubernamentales (Lessig, 1999).

En esta línea, la identificación del código tecnológico con los códigos corporales normativos -ya sean de género, sexuales, raciales o de otra índole- puede resultar más fluida y clara. Al igual que el establecer un paralelismo entre las funciones $y$ dinámicas del código tecnológico y la performance, en el sentido de corporeización de los códigos culturales, es decir, de repetición estilizada de actos instituidos en el cuerpo, a través de la cual la identidad es constituida, codificada y naturalizada (Butler, 1998[1990]).

Del mismo modo que la efectividad de los modelos de regulación identitaria-corporal y de control social residen en su 
pretendido carácter natural $e$ invariable, la funcionalidad del código, de la sofisticación tecnológica, en general, tiene que ver con hacerlo invisible (Thomas, 2005).

El funcionamiento de la performance en relación al cuerpo y del código tecnológico en relación a la máquina comparten como rasgo definitorio la repetición.

Así mismo, mediante la performance se puede emplear la repetición con diferencias (Thomas, 2005) y modificar y transgredir los códigos corporales normativos (Butler, 2007 [1989], 2002[1993]), y en el plano de la codificación tecnológica, el código cerrado puede ser alterado mediante las prácticas hackers, el open source y a través del software libre.

La cultura hacker y la filosofía y movimiento del open source y del software libre se basan en abrir los códigos para modificarlos, distribuirlos y compartirlos, evidenciando, de este modo, la función normativa del código y la estrecha conexión entre ley, industria y capital (Himanen, 2004[2001]; Thomas, 2002).

Por esta razón, el software libre y la performatividad se convierten en herramientas mediante las que desestabilizar, romper y subvertir códigos reguladores, uno en el plano tecnológico, y otro en el plano corporal.

Código, performance y cuerpo son ámbitos conectados en el marco de la tecnocultura, donde la codificación cultural del cuerpo funciona en términos abstractos aplicándose a todos los cuerpos. Por su parte, la performance es cuerpo en sí misma, es material, carne, representación y práctica particular que produce, reproduce o transgrede código cultural (Thomas, 2005). Desde una lectura feminista hacker basada en hacer visible el código fuente con objeto de alterarlo, el cuerpo transforma el código cultural en performance, del mismo modo que el acto performativo traduce el código en cuerpo, con la potencialidad, ambos, de alterar el código normativo en corporalidades, subjetividades, imaginarios, representaciones y prácticas transgresoras.

Ineludiblemente, las producciones, materialidades, performatividades, prácticas y vivencias corporales en torno a codificaciones normativas de género, sexualidad, raza, etc. se 
desarrollan en dimensiones diferenciadas a la codificación cerrada de los productos y servicios tecnológicos, como el software privativo. Pero establecer una articulación entre cuerpo y tecnología mediante la noción de código nos permite habilitar la figura hacker como un espacio de resistencia para los feminismos.

En acuerdo con Daniela De Araujo (2018), el término hacker no siempre ha de estar vinculado a la tecnología o al movimiento hacker, sino que, como realizo en este trabajo, puede ser apropiado para activar otras críticas e iniciativas que apuestan por transformar un sistema o por el conocimiento libre.

Los feminismos como prácticas hackers: abrir los códigos de sistemas operativos culturalmente hegemónicos

Las herramientas tecnológicas comenzaron a funcionar como cajas negras donde se desconoce el código de los programas, suscritos a patentes y beneficios, tras la década de los setenta. A partir de aquí, nos convertimos en consumidorxs dependientes de actualizaciones compatibles con nuestro hardware. Así mismo, las tecnologías como cajas negras implican una invasión en nuestra privacidad y seguridad digital, y el establecimiento de mecanismos de control (Soria, 2016).

El código cerrado por sistemas de regulación hegemónicos lo encontramos tanto en el plano tecnológico como en el plano corporal. Por lo que repensar los cuerpos y las tecnologías desde perspectivas feministas hacker dirigidas al código abierto nos permite explorar, descodificar, modificar y reapropiarnos de nuestros cuerpos y máquinas.

Abrir la caja negra de la tecnología ha sido una tarea de las prácticas hacker, mientras que descodificar la caja negra del cuerpo ha sido una lucha, históricamente, feminista (Soria, 2016).

En este sentido, el software libre, además de transformar las tecnologías nos permite desarrollar lecturas sobre los feminismos como narrativas y prácticas dirigidas a comprender, intervenir y subvertir sistemas operativos culturalmente hegemónicos (Egaña, 2017). 
La cultura hacker, open source y el software libre pueden ser aliados de las luchas feministas. No obstante, es necesario considerar que la ética y política emancipatoria que orienta tales escenarios no incluye la crítica feminista, a pesar de que, históricamente, los feminismos han desarrollado una construcción colectiva del conocimiento cuestionando cómo este ha sido producido para excluir a otrxs (Tejidos hackfeministas, 2014).

En concreto, el software libre es más que una tecnología alternativa, es una filosofía y cultura que se organiza en torno a principios políticos orientados a la emancipación y autonomía para generar espacios de participación y producción de conocimiento horizontales, igualitarios y anticapitalistas, pero esta construcción colectiva no sucede en términos de ideologías y políticas feministas (Ortman, 2016).

La cultura hacker y software libre no se traduce para, ni incorpora a las mujeres y diversidades de género, sexuales y corporales, donde múltiples trayectorias y experiencias son relegadas, invisibilizadas u objeto de sexismo, racismo y lgtbiqfobia. Tales escenarios no se constituyen como espacios seguros donde se cuestionen jerarquizaciones de poder en torno al género, la raza, la clase, la diversidad funcional, la sexualidad, etc. (Toupin, 2014).

Por ello, feministas y disidencias de género, sexuales, migratorias y corporales han tenido que desplegar mecanismos de (auto)inclusión u organizarse autónoma y colectivamente para desarrollar colectivamente tecnologías libres e intervenir la maquinaria tecnocultural normativa desde perspectivas feministas (Toupin, 2014).

En esta línea, los hackfeminismos hackean cuerpo, género, sexualidad y también tecnología. Desarrollan tecnologías libres y espacios de seguridad para potenciar la diversidad, las disidencias y las resistencias (Briones, 2016).

Interpretar los feminismos desde una perspectiva hacker implica hackear el sistema cisheteronormativo para acceder, modificar y reconfigurar su código fuente, aquello que Butler (2007[1989]) denominó la matriz heterosexual. 
En este sentido, los fallos en el sistema hetero-blancopatriarcal, desde la perspectiva de las tecnologías libres, son espacios de intervención para llegar a los códigos. De este modo, ciertos feminismos y trayectorias corporales no normativas funcionan como un virus que pueden ser interpretados en términos de reprogramación de los códigos dominantes (Egaña, 2015 y 2017).

No obstante, igualmente, se requiere generar herramientas tecnológicas libres desde prácticas feministas autónomas, colectivas y anticapitalistas.

La apropiación tecnológica no se reduce al manejo de diferentes dispositivos generados por corporaciones industriales y empleados como mecanismos de control, más bien implica abrir los códigos, conocer su funcionamiento, intervenirla y generar herramientas propias a través de lo colectivo (Soria, 2016).

Por tanto, una tecnología feminista será una tecnología anticapitalista, basada en deconstruir las maquinarias y hacerlas funcionar en el sentido opuesto para el que fueron diseñadas. Así mismo, una tecnología feminista se orienta a crear espacios de seguridad en la calle y en la red. De igual forma, una relación feminista con la tecnología incluye el cuerpo y la subjetividad e implica hackear no sólo el código fuente del hardware y software sino, también, del cuerpo, la historia y la maquinaria cultural. Relacionarnos con las tecnologías desde perspectivas feministas es un ejercicio colectivo de explorar los cuerpos y las máquinas, de reciclar artefactos tecnológicos para resistir a la obsolescencia programada, y programar la obsolescencia de modelos corporales y culturales normativos (Egaña, 2013).

En este último sentido, las intersecciones entre cultura hacker y feminismos pueden ser múltiples y fructíferas. Por un lado, el software, el hardware y las herramientas libres son mecanismos para la autonomía tecnológica feminista, en especial, para trabajar políticamente temas de autodefensa digital frente a las ciberviolencias (De Araujo, 2018). Por otro lado, los feminismos se tornan enfoques y prácticas críticas frente a la masculinización 
blanca cisnormativa y contra las relaciones de poder presentes en la cultura y espacios de experimentación hacker.

Además, en la relación entre tecnologías y feminismos, Haraway (1995[1991]) nos advertía de la urgencia feminista de intervenir la tecnociencia y la codificación para reinventar nuestros cuerpos mediante la imaginería cyborg como política contra el código único. Así mismo, Preciado (2008) compartía su experiencia de hackear el género como usuario copyleft de la testosterona para abordar los cuerpos como laboratorios políticos y potenciales espacios de resistencia a la normalización, y para interpretar diversas micropolíticas de género y sexuales como prácticas hacker y modos de producción de saberes y transformación.

En este contexto de conexión entre feminismos y tecnologías a continuación comparto algunas reflexiones derivadas de mi proyecto de investigación donde abordo la perspectiva hacker como un espacio de resistencia feminista para abrir y subvertir cualquier sistema codificado, ya sea el software, el hardware, el cuerpo o la producción hegemónica de conocimiento. Para ello he desarrollado trabajo de campo desde una metodología etnográfica feminista en la escena transfeminista de Barcelona, donde como investigadora y activista transfeminista he participado en talleres, jornadas y he realizado entrevistas a colectivos y trayectorias que abordan la conexión entre disidencias sexuales, corporales y tecnológicas desde perspectivas (trans)feministas.

En este sentido, los transfeminismos pueden ser interpretados como la materialización local de las teorías y movimientos queer, los cuales se enunciaron por primera vez como alianzas transfeministas en las Jornadas Feministas Estatales de Granada en 2009, en el Manifiesto para la Insurreción Transfeminista $^{1}$ (2010) y en el Manifiesto TransfeministaTransfronterizo $^{2}$ (2010). Las redes transfeministas se tejen a lo

\footnotetext{
${ }^{1} \mathrm{http}: / /$ ideadestroyingmuros.blogspot.com/2009/12/manifiesto-para-lainsurreccion.html

${ }^{2}$ https://sindominio.net/karakola/IMG/pdf_Manifiestofinal2.pdf
} 
largo de la geografía de todo el estado y articulan diversas luchas en torno a la postpornografía, trabajo sexual, despatologización trans*, contra la normalización de los movimientos feministas y lgtb, las resistencias migrantes y racializadas, la diversidad funcional, software libre y tecnologías autónomas, etc. (Egaña y Solá, 2016).

En concreto, a continuación comparto las reflexiones derivadas de una parte de la experiencia etnográfica a partir de los diálogos con Alex Hache, Lucía Egaña, Klau Kinki, Ce, Kina, Elena Urko y Diego Marchante. Reflexiones que he organizado bajo las siguientes enunciaciones que sintetizan las aportaciones $e$ interpretaciones de cada una de las entrevistas.

Claves feministas para la (auto)inclusión, autonomía y emancipación tecnológica

La entrevista con Alex Hache $e^{3}$ investigadora y tecnoactivista, donde me compartía su trayectoria y el trabajo de Donestech, me proporcionó claves para abordar la autonomía tecnológica feminista.

En 2006, surgiría Donestech ${ }^{4}$ como espacio que propiciaría una red y la creación de eventos donde se empezó a trabajar el acceso y desarrollo de tecnologías libres desde perspectivas feministas (Alex, 2017).

Donestech es un proyecto de ciberfeminismo de investigación que aborda las desigualdades en la relación género y tecnología a través de la investigación, divulgación, producción audiovisual y formación.

En relación con los proyectos de investigación, su trabajo supera el enfoque de la exclusión centrado en la brecha digital de género, $e$ indaga en las trayectorias, subjetividades, deseos, formas de pensar y de desarrollar tecnologías por parte de mujeres y otras diversidades con el objetivo de propiciar referentes y claves de emancipación (Alex, 2017). En este ámbito, destacan los proyectos

3 [Alex Hache, entrevista persona, 14 de junio de 2017, Barcelona] .

4 https://www.donestech.net/ 
de investigación LeLArt y LeLaCoders sobre las experiencias y trayectorias con TIC de tecnoartistas y de mujeres desarrolladoras de software libre y/o hackers (Vergés; Hache; Cruells, 2014).

Por otro lado, el proyecto de Donestech me ha facilitado conocer el trabajo con tecnologías libres desde el paradigma de (auto)inclusión. En este sentido, desarrollan talleres de formación en herramientas libres para colectivos de mujeres, feministas y lgtbq, tanto en producción audiovisual, streaming como en software libre u otras herramientas alternativas, prestando más énfasis, en los últimos años, en temas de privacidad, seguridad y autodefensa digital (Alex, 2017).

Considero que el trabajo de Donestech materializa la conexión entre feminismos y hacking al abordar tanto la visibilización como la (auto)inclusión y formación de mujeres, feministas y diversidades en el ámbito de las tecnologías libres. Así mismo, operan la articulación feminismos y hacking como herramienta contra las violencias digitales mediadas por las TIC.

Por último, en relación con el hacking contemporáneo y el cuerpo, recojo una reflexión que Alex me compartía:

Las nuevas prácticas de hacking están saliendo, obviamente, del ordenador y se están expandiendo en lo biológico, en el cuerpo, en los biolabs, en todo lo que ya sabemos desde hace mucho tiempo, en el trabajo con plantas, saberes ancestrales, permacultura. El hacking contemporáneo se está dando, no tanto en el código del software sino que está tocando el hardware, los cuerpos, los fluidos, lo orgánico. (Alex, 2017).

\section{Hackear el cuerpo como dispositivo codificado}

Los diálogos y encuentros con Lucía Egaña han sido muy inspiradores en mi trabajo y en el desarrollo de lecturas que conectan cuerpo, tecnología y código, así como en la interpretación de los feminismos como prácticas hacker. 
Desde su trayectoria migrante, postpornográfica, disidente sexual y tecnológica forma parte de proyectos como la Muestra Marrana y colectivos como Minipimer.tv, desBASURAment, Real Archivo Sudaca y Cooptecniques, entre otros ${ }^{5}$.

En la entrevista ${ }^{6}$, Lucía me compartía cómo en su trayectoria feminista, postpornografía y software libre se fueron entrelazando de forma simultánea. En este sentido, me trasladó un paralelismo entre el software libre y el cuerpo, a través del cual, el software libre como acceso al código, le ha permitido pensar el cuerpo, la sexualidad y la subjetividad como codificación (Lucía, 2017).

En relación a la articulación feminista con las tecnologías, Lucía me ofreció perspectivas críticas que exceden el acceso a las tecnologías hegemónicas. Destaca cómo el hackfeminismo da lugar a otras articulaciones que tienen que ver con el cuerpo, donde se cruzan las tecnologías de producción y representación del género, la sexualidad y la raza, y la noción de tecnología es ampliada para relacionarla con otros ámbitos. Así mismo, otro nivel de articulación con las tecnologías es imaginar y construir otras formas feministas de pensar y relacionarse con las tecnologías. En esta línea, incorpora reflexiones sobre el ensayo/error, los procesos y el fracaso como lugares para reconvertir la rabia en terrenos fértiles, en disidencias (Lucía, 2017).

Las percepciones de Lucía sobre la conexión entre transfeminismos y tecnologías fueron muy reveladoras para entender dichas articulaciones como un escenario propiciado por ciertos agentes y espacios de activación que están desarrollando redes y proyectos muy fructíferos, a la par que cuestionan las escenas normativas del hacking y el software libre (Lucía, 2017).

Por otro lado, las reflexiones compartidas me permiten romper las concepciones y representaciones tradicionales de la tecnología, abordar el cuerpo y la sexualidad como tecnología y considerar la matriz heteronormativa como el código fuente de la

${ }^{5}$ http://www.lucysombra.org/

6 [Lucía Egaña, entrevista personal, 16 de febrero de 2017, Barcelona] 
codificación corporal-sexual. Donde, hackear dicha matriz desde trayectorias de disidencia corporal-sexual es un complejo y lento proceso. En este sentido, Lucía establecía: "El postporno o el software libre no van a liberar tu cuerpo y la tecnología de forma completa, pero son procesos de hackeo y deconstrucción" (Lucía, 2017).

El cuerpo es una tecnología. Entonces, la tecnología disidente es nuestra propia existencia, directamente ${ }^{7}$

Los diálogos con Klau Kinki ${ }^{8}$, feminista y disidente sexual post-porno, me permitieron conocer sus experiencias vinculadas, por un lado, con espacios y redes del feminismo postpornopunk y, por otro lado, con su participación en diferentes escenarios hackers. Experiencias inmersas en dos enclaves aparentemente desconectados entre sí, entre el espacio normativo hacker y del software libre, y el espacio feminista de disidencia sexual que continuaba empleando sistemas operativos privativos (Klau, 2017). $\mathrm{Su}$ trayectoria y trabajo es un referente disidente de conexión crítica de ambos ámbitos.

Klau me trasladó como dicha articulación se inició con su trabajo en Generatech ${ }^{9}$, donde abordaba la tecnología más allá de su relación con el género, realizando talleres, charlas y performances, así como, potenciando espacios de conexión entre colectivos transmaricabollo y hackers. Desde su experiencia, Generatech surgió como un proyecto para generar contaminaciones. En este sentido, la vinculación era el código:

El código privativo y libre, el código de la máquina y del cuerpo, el código del heteropatriarcado y del feminismo. Por lo que la contaminación se dirigía a abrir los códigos, entender cómo funcionan las herramientas, las máquinas,

7 [Klau Kinki, entrevista personal, 1 de marzo de 2017, Barcelona]

8 [Klau Kinki, entrevista personal, 1 de marzo de 2017, Barcelona].

9 http://generatech.ningunlugar.org/ 
los cuerpos, el heteropatriarcado y los feminismos para generar nuestro propio contenido (Klau, 2017).

Desde la conexión entre máquina y cuerpo, código y performance me compartía su experiencia en el proyecto Mutanger. Laboratorio Tóxico de Alta Tensión ${ }^{10}$, un espacio de experimentación técnica con herramientas electrónicas, mecánicas y tecnológicas y de experimentación corporal con performance.

Así mismo, Klau me trasladaba el proceso de Pechblenda ${ }^{11}$, como un espacio transhackfeminista que emergió ante la normatividad de los hacklabs de su contexto y desde la disidencia y rabia. Un laboratorio de experimentación bio-electro-química y de hardware libre, de aprendizaje, autoformación y generación de conocimientos libres (Klau, 2017).

Precisamente, la vinculación entre transfeminismo y hacker a través del término transhackfeminismo se acuño con Pechblenda (Kinki, 2013) y, posteriormente, se empleó para nombrar diferentes encuentros transhackfeministas ${ }^{12}$ (Klau, 2017).

Igualmente, Klau me compartió el proceso de Gynepunk ${ }^{13}$, un proyecto que surgió como un tentáculo de Pechblenda y es consecuencia del trabajo conectado entre cuerpo, tecnología, biohacking y transfemininismo. Gynepunk es un laboratorio de biohacking dirigido a cuestionar y hackear el cuerpo, la biomedicina y la ginecología, así como las tecnologías y metodologías de diagnóstico y tratamiento médicas a través de la autoexploración, la experimentación, el desarrollo de técnicas ginecobstétricas, prostático degeneradas autogestionadas y diagnósticos autónomos desde conocimientos compartidos experimentados desde cada cuerpo (Klau, 2017).

\footnotetext{
${ }^{10} \mathrm{https} / /$ mutangerlab.wordpress.com/

${ }^{11} \mathrm{https} / /$ network23.org/pechblendalab/trans-hack-feminista/

12 https://transhackfeminist.noblogs.org/

${ }^{13}$ https://gynepunk.hotglue.me/?intro; http://gynepunk.tumblr.com/
} 
Laboratorio de investigación y experimentación sobre cuerpos, sexualidades, subjetividades y tecnologías

Las entrevistas $y$ encuentros con $\mathrm{Ce}$ y $\mathrm{Kina}^{14}$, me permitieron conectar con el proyecto de Quimera Rosa ${ }^{15}$, un laboratorio de investigación y experimentación que emergió como un trabajo sobre el propio cuerpo, la propia sexualidad y subjetividad. Donde, inicialmente, predominaba la performance y las prácticas postpornográficas como herramientas feministas para generar otras ficciones, imaginarios, representaciones y prácticas sexuales (Kina, 2017) que, además, desarticulaban las fronteras entre lo público y lo privado (Ce, 2017).

Conocer el trabajo de Quimera Rosa me ha permitido abordar cuestiones en torno al género, la sexualidad, el cuerpo y las tecnologías desde los diversos proyectos que desarrollan. Donde los ejes transversales en todos ellos son la deconstrucción de la identidad, las tecnologías de producción de subjetividad y la dimensión colectiva del trabajo. Por su parte, las herramientas para abordar tales cuestiones han ido mezclándose y mutando desde prácticas postpornográficas, prótesis sexuales, BDSM, dispositivos tecnológicos, sonido, texto, performance, talleres, instalaciones, biología y biohacking (Ce, 2017).

El trabajo de Quimera Rosa se materializa en proyectos transdisciplinares que conectan, re-apropian y contagian los ámbitos artísticos, hackers y, más recientemente, el ámbito de la ciencia biomédica, a partir del proyecto de Transplant ${ }^{16}$ (Ce, 2017).

Las entrevistas con Quimera Rosa, me han permitido conectar las prácticas hacker y los transfeminismos, al compartirme cómo, desde su trayectoria, se ha producido un diálogo y contaminación mutua. Lo que desde el trabajo de Quimera se

\footnotetext{
${ }^{14}$ [Ce, entrevista personal, 28 de marzo de 2017, Barcelona] [Kina, entrevista personal, 28 de marzo de 2017, Barcelona].

${ }^{15} \mathrm{http}: / /$ quimerarosa.net/

${ }^{16} \mathrm{http}: / /$ quimerarosa.net/transplant/
} 
aplica al género, la sexualidad, el cuerpo y la subjetividad, desde lo hacker se aplica a las máquinas y la tecnología (Kina, 2017).

Así mismo, su trabajo con tecnología y sonido viene de las colaboraciones en encuentros hackers y de open source. En este sentido, los dispositivos que producen y todo su trabajo parte de la filosofía Do it Togethery Do It With Other que se basan en la idea de generar en colectivo a partir de herramientas y conocimientos libres. En consecuencia, lo que producen es compartido y con licencia abierta para estudiar y modificar (Ce, 2017).

En esta línea, Quimera Rosa conecta hackeo corporal y tecnológico a través del biohacking desde la idea de abrir la caja negra del cuerpo, saber cómo funciona y darle otras utilidades diferentes para las que está diseñada (Kina, 2017).

El proyecto Transplant, por ejemplo, tiene que ver con un proceso de biohacking, de conectar disciplinas, reapropiarse de herramientas biomédicas y técnicas, entre otras, desvelar cómo funcionan y modificarlas para producir subjetividad desde la experimentación corporal (Kina, 2017).

Desde mi punto de vista, la trayectoria de contagio entre transfeminismos y hacking de Quimera Rosa está permeada por el desarrollo de talleres que activan espacios colectivos, a modo de micro-laboratorios políticos nómadas, para hackear modelos identitarios dominantes. Así como, por la autoexperimentación a través de la performance o el biohacking donde producen otros códigos corporales y subjetividades.

\section{Hacking corporal desde imaginarios y prácticas postpornográficas}

Elena Urko es activista postporno y unx de lxs impulsorxs del colectivo Post-op. Su trayectoria se despliega tanto en contextos feministas autogestionados como en espacios institucionales académicos y artísticos, a través de una polisemia de trabajos: desde performances, instalaciones y producción audiovisual hasta charlas y desarrollo de talleres. 
A partir de la entrevista con Elena Urko ${ }^{17}$ pude compartir su trabajo en Post-op $^{18}$, un grupo interdisciplinar de experimentación e investigación sobre cuerpo y postpornografía. Post-op es un proyecto, que aborda la sexualidad y el género desde el cuerpo y la performance, orientado a generar imaginarios, representaciones y prácticas no normativas, así como a la sexualización y okupación del espacio público (Urko, 2017).

Post-op fue uno de los primeros proyectos que empezó a activar prácticas postpornográficas y emergió como un grupo de performers con la inquietud de llevar al espacio público aquello que estaban vivenciado en el contexto transmaricabollo de Barcelona. Posteriormente, se sumergieron en las tecnologías de producción audiovisual desarrollando vídeos que compartían en diferentes jornadas, así como en su web-blog (Urko, 2017).

Desde mi punto de vista, el trabajo de Post-op (2013) con performance y producción audiovisual ha sido un referente crucial, a nivel personal y colectivo, en redes (trans)feministas para generar "otras" representaciones que nos han permitido ampliar imaginarios, deseos y prácticas sexuales. Descodificando y hackeando, de esta forma, las construcciones normativas de cuerpo y sexualidad, y reprogramando códigos alternativos.

Además, más allá de la importancia de la representación, como me compartía Urko, su trabajo con postpornografía constituye herramientas que pasan por el propio cuerpo y reflejan los procesos personales (Urko, 2017). En este sentido, interpreto la postpornografía junto con Preciado (2008) como una autoexperimentación política de reprogramación del propio cuerpo.

Post-op inició su trayectoria trabajando con performance y vídeo, también produjo la Revista Piratte y, posteriormente, empezaría a desarrollar talleres. Urko identificaba la realización de talleres como un punto de inflexión en la trayectoria de Post-op, donde se ha sucedido una mutación desde los primeros talleres,

\footnotetext{
17 [Elena Urko, entrevista personal, 18 de octubre de 2017, Barcelona].

${ }^{18} \mathrm{http} / / /$ postop-postporno.tumblr.com/; http://postporno.blogspot.com/
} 
más enfocados en la representación, en generar un porno propio por parte de lxs participantes en los talleres recientes, orientados a la experimentación corporal desde prácticas postpornográficas, con más énfasis en las experiencias y procesos, así como en ejercicios de desinhibición, confianza y límites (Urko, 2017).

En líneas generales y en la actualidad, Urko desarrolla talleres de BDSM y diversos talleres corporales ${ }^{19}$ que interpreto con dispositivos políticos al generar ejercicios colectivos de reprogramación de deseos, placeres y prácticas sexuales.

Así mismo, Urko, me compartía la participación en el proyecto de Yes We Fuck ${ }^{20}$ como otro punto de inflexión en el trabajo de Post-op, a partir del cual empezaron a desarrollar talleres de postpornografía para personas con diversidad funcional generando espacios para trabajar con diversidades corporales mediante ejercicios, dinámicas y lugares accesibles (Urko, 2017).

Derivado de estos talleres de postpornografía para personas con diversidad funcional-corporal y de los encuentros en eventos sobre tecnología y hacking emergería Pornotopedia ${ }^{21}$. Un proyecto articulado en torno a la idea de autoconstruir, reciclar o modificar prototipos, prótesis, ortopedia y juguetes sexuales para que estos sean más cercanos a los intereses, deseos, prácticas sexuales, corporalidades, movilidades y sensitividades de cada unx (Urko, 2017).

Considero que el trabajo de Post-op con talleres para personas con diversidad funcional-corporal y con el proyecto de Pornotopedia nos ha interpelado a todxs porque han generado alianzas entre diversos cuerpos y luchas y han incluido la normalidad corporal y la capacidad como ejes de poder en las políticas (trans)feministas.

La vinculación del trabajo de Post-op entre postpornografía y tecnología se desarrolla a diferentes niveles. Además de la relevancia de las tecnologías del cuerpo, del género y de la

\footnotetext{
${ }^{19} \mathrm{https}: / /$ elenaurkopostop.com/

${ }^{20} \mathrm{http}: / /$ www.yeswefuck.org/

${ }^{21} \mathrm{http}: / /$ postop-postporno.tumblr.com/Pornortopedia
} 
sexualidad, desde la perspectiva de Urko, la imbricación del postporno con la tecnología también se relaciona con las prótesis empleadas en las diferentes prácticas, talleres, performances y vídeos, así como con las tecnologías para la producción audiovisual y las tecnologías de la representación (Urko, 2017).

\section{Cuerpo-archivo contra-hegemónico desde lo analógico y digital}

Diego Marchante, Gender-Hacker ${ }^{22}$ es activista transfeminista, docente, investigador y artista transmedia. En la entrevista ${ }^{23}$, Diego me hizo partícipe de su trayectoria y trabajo en torno a hackear cuerpo, género, sexualidad y archivo.

En relación a su trabajo de investigación, hablábamos de Archivo $T$, un proyecto que se materializa en un archivo digital ${ }^{24} y$ en toda una serie de exposiciones, como Transcyorbollera.Transcyborgllera. Hackeando el archivo desde una perspectiva transfeminista y queer. Así mismo, fue parte de su proceso de investigación doctoral (Marchante, 2015).

Archivo $T$ es un trabajo que recoge y articula las genealogías de los movimientos feministas, lgtb, queer y trans* en las últimas décadas. Así mismo, es presentado de forma cronológica y temática a través de herramientas abiertas de visualización interactiva, de gestión de constelaciones y gestión de timeline.

Diego me compartía que Archivo $T$ es un proyecto de hackear el archivo hegemónico, un contra-archivo político y artístico que, no solo aborda las genealogías ausentes en la historiografía dominante, sino que además, se desarrolla y presenta a través de formatos que exceden las maneras clásicas de archivar, en este sentido, las herramientas digitales ofrecen múltiples potencialidades (Diego, 2017).

Igualmente, me trasladaba como Archivo $T$, también, es cuerpo, somáteca, es su propio cuerpo, su trayectoria transfeminista, bollera y trans*, su cuerpo-archivo derivado de las

\footnotetext{
${ }^{22} \mathrm{http}: / /$ genderhacker.net

23 [Diego Marchante, entrevista personal, 23 de octubre de 2017, Barcelona].

${ }^{24} \mathrm{http}: / /$ genderhacker.net/?portfolio=archivo-t
} 
luchas y referentes de disidentes corporales, de género y sexuales (Diego, 2017).

Por otro lado, Diego me compartía su trayectoria corporal como una experiencia y proceso de hackear el género mediante tecnologías de des-identificación, de lenguaje, materiales, hormonales, digitales y virtuales. En este último sentido, destaca su trabajo con performances, fotografía y vídeo (Diego, 2017).

La idea de hackear el cuerpo además de aplicarla en su trayectoria personal, la desarrollaba en talleres como GenderhacKing. Inversión performativa y subversión de la masculinidad. Respecto a este proyecto, Diego me compartía la estructura y proceso de los talleres basados en la metodología Drag-King pero, en este caso, se articulaban en torno a una comprensión del cuerpo como un hardware a hackear mediante la instalación de softwares alternativos a la hegemonía heteronormativa, que se desarrollaban empleando diversas tecnologías del género analógicas y digitales (Diego, 2017). En este sentido, el dispositivo Drag-King funciona como una reprogramación colectiva del género que además interviene el espacio público.

\section{Reflexiones finales}

Las perspectivas críticas feministas han incidido en los ámbitos tecnológicos del mismo modo que los feminismos han mutado en dicho proceso. En este sentido, las aproximaciones feministas a la tecnología se han transformado desde corrientes tecnofóbicas hasta perspectivas que apuestan por la subversión de la tecnocultura dominante mediante la reconfiguración y reapropiación tecnológica.

A partir de la década de los noventa, en un contexto de desarrollo de la cultura digital, el trabajo de Haraway inauguró perspectivas posfeministas hacia las tecnologías mediante la propuesta cyborg, influyendo en el desarrollo de nuevas posibilidades de análisis y prácticas políticas feministas orientadas a la transformación radical de la tecnocultura hegemónica. 
En este marco, desde ciertas perspectivas y prácticas feministas se potencian fructíferas articulaciones que abordan las múltiples dimensiones de las tecnologías. En esta línea, en mi trabajo sitúo en un mismo plano cuerpo y tecnología e interpreto una conexión entre códigos tecnológicos y códigos corporales, así como entre feminismos y hacking.

En este sentido, recojo las reflexiones derivadas de una parte de mi proceso etnográfico a través de los diálogos con Alex Hache, Lucia Egaña, Klau Kinki, Ce, Kina, Elena Urko y Diego Marchante como trayectorias que conectan críticamente cuerpos, tecnologías, feminismos y hacking. Interpreto las experiencias y proyectos compartidos como prácticas feministas orientadas a: abrir y desvelar los códigos tecno-culturales de programación corporal y subjetiva; producir códigos alternativos; y, además, hackear los mecanismos dominantes de construcción y validación de conocimiento generando conocimientos de resistencia basados en trayectorias disidentes, experiencias colectivas $y$ experimentaciones que operan en el propio cuerpo.

\section{Referencias bibliográficas}

ALEXANDER, Jonathan. Queer Webs: Representations of LGBT People and Communities on the World Wide Web. International Journal of Sexuality and Gender Quarterly, vol.8, n.2-3, 2002, pp.77-84. DOI:10.1023/A:1015821431188.

BRIONES, Patricia. Hagámoslo juntas (DIT): apuntes para reflexionar en torno al hackfeminismo. En: SORIA, Irene (Coord.). Ética Hacker, seguridad y vigilancia. México D.F, Universidad del Claustro de Sor Juana, 2016, pp.217-253.

BoIX, Montserrat. Desde el Ciberfeminismo hacia la Tecnopolítica feminista. Revista Pillku, $\mathrm{n}^{\circ} \quad 18,2015$ [http://www.pillku.com/article/desde-el-ciberfeminismo-hacia-latecnopolitica-fem/ - consultado en: 23 may.2018].

BRAIDOTTI, Rosi.Cyberfeminism with a difference. 1996. [http://webs.ucm.es/info/rqtr/biblioteca/ciberespacio\%20gltb/Cyberfem 
inism\%20with\%20a\%20difference.pdf - consultado en: 5 may. 2018].

BRYSON, Mary. When Jill Jacks In: Queer Women and the Net. Feminist Media Studies, vol. 4, n³, 2004, pp.239-254. DOI: $10.1080 / 1468077042000309928$.

BUTLER, Judith. Actos performativos y constitución del género: un ensayo sobre fenomenología y teoría feminista. Debate Feminista, n ${ }^{\circ} 18$, 1998[1990], pp.296-314. [http://www.debatefeminista.cieg.unam.mx/wpcontent/uploads/2016/0 3/articulos/018_14.pdf - consultado en: 15 jul.2018].

BUTLER, Judith. Cuerpos que importan. Sobre los límites materiales y discursivos del "sexo". Buenos Aires, Paidós, 2002(1993).

BUTLER, Judith. El género en disputa. El feminismo y la subversión de la identidad. Barcelona, Paidós, 2007(1989).

CARLSON, Dennis. Gay, Queer, and Cyborg: The Performance of Identity in a Transglobal Age. Discourse: Studies in the Cultural Politics of Education, vol.22, $\quad \mathrm{n}^{\circ} 3, \quad 2001, \quad$ pp.297-309. DOI: $10.1080 / 01596300120094343$.

CODE, Lorraine. What Can She Know? Feminist Theory and the Construction of Knowledge. New York, Cornell University Press, 1991.

CockBURN, Cynthia. Brothers: Male Dominance and Technological Change. Londres, Pluto Press, 1983.

DANIELS, Jessie. Cyber Racism: White Supremacy Online and the New Attack on Civil Rights. New York, Rowman \& Littlefield Publisher, 2009a.

DANIELS, Jessie. Rethinking cyberfeminism(s): Race, gender and embodiment. WSK's Women's Studies Quarterly, vol.37, nº.1\&2, 2009b, p.101-124. DOI:10.1353/wsq.0.0158

De ARAujo, Daniela. Feminismo e Cultura Hacker: Intersecçóes entre Política, Gênero e Tecnologia. Tesis doctoral. Campinas, Universidade Estadual de Campinas, 2018.

De LAURETIS, Teresa. Technologies of Gender. Essays on Theory, Film and Fiction. London, Macmillan Press, 1989. 
De Miguel, Ana; BoIX, Montserrat. Los géneros de la red: los ciberfeminismos. En: NATANSOHL, Graciela (coord.). Internet en código femenino. Teorías y prácticas. Buenos Aires, La Crujía Ediciones, 2013, pp.37-73.

EGAÑA, Lucía. Tecnotransfeminismo: Apuntes para una tecnología transfeminista. En: SOLÁ, Miriam; URKO Elena (coords.). Transfeminismos. Epistemes, fricciones y flujos. Tafalla Nafarroa, Txalaparta, 2013, pp.313-324.

EGAÑA, Lucía. Trincheras de carne. Una visión localizada de las prácticas postpornográficas en Barcelona. Tesis doctoral. Barcelona, Universitat Autònoma de Barcelona, 2015.

EGAÑA, Lucía. Hacking feminista: reapropiando códigos, desprogramando cuerpos. Oficina Antivigilancia, Boletín\#15, 2017. [https://antivigilancia.org/es/2017/05/hacking-feminista-2 -consultado en: 24 jul. 2018].

EGAÑA, Lucía; SolÁ, Miriam. Hacking the body. A Transfeminist War Machine. TSQ: Transgender Studies Quarterly, vol.3, n'.1-2, 2016, pp.74-80. DOI:10.1215/23289252-3334223.

FERNÁNDEZ, Maria. Cyberfeminism, Racism, Embodiment. In: FERNÁNDEZ, María; WILDING, Faith; WRIGHT, Michelle M (eds.). Domain Errors! Cyberfeminist Practices. New York, Autonomedia, 2002, pp.29-44.

Fernández, María; WiLding, Faith; Wright, Michelle M (eds.). Domain Errors! Cyberfeminist Practices. New York, Autonomedia, 2002.

GAJJALA, Radhika. "Third World" perspectives on cyberfeminism. Development in Practice, vol. 9, $\mathrm{n}^{\circ} .5$, 1999, pp.616-619. DOI:10.1080/09614529952774.

GALPIN, Vashti. Women in Computing around the World. SIGCSE Bulletin, vol. $\quad 34, \quad \mathrm{n}^{\circ} . \quad 2, \quad 2002, \quad$ pp.94-100. DOI: $10.1145 / 543812.543839$.

GILL, Rosalind. Review: Technofeminism. Science as Culture, vol.14, $\mathrm{n}^{\circ}$. 1, 2005, pp.97-101. DOI:10.1080/09505430500042130.

GRINT, Keith; GILL, Rosalind (ed.). The Gender-Technology Relation: Contemporary Theory and Research. London, Taylor and Francis, 1995. 
GonZÁlEZ, Marta; PÉREZ, Eulalia. Ciencia, Tecnología y Género. Revista Iberoamericana de Ciencia, Tecnología y Sociedad-CTS, n.2, 2002. [https://www.oei.es/historico/revistactsi/numero2/varios2.htm consultado en: 26 jul. 2018].

GONZÁLEZ, Marta; FERNÁNDEZ, Natalia. Ciencia, tecnología y género: Enfoques y problemas actuales. Revista iberoamericana de ciencia tecnología y sociedad, vol.11, $\mathrm{n}^{\circ} .31,2016, \quad$ pp.51-60. [http://www.scielo.org.ar/scielo.php?script $=$ sci arttext\&pid $=\mathrm{S} 185000$ $132016000100004 \& \operatorname{lng}=e s \& \operatorname{lng}=e s$ - consultado en: 25 may. 2018].

HARAWAY, Donna. Situated knowledges: The science question in feminism and the privilege of partial perspective. Feminist studies, vol.14, nº.3, 1988, pp.575-599. DOI:10.2307/3178066.

HARAWAY, Donna. Ciencia, cyborgs y mujeres. La reinvención de la naturaleza. Madrid, Cátedra, 1995 (1991).

HARAWAY,_Donna._Modest_Witness@Second_Millenium. FemaleMan_C.Meets_OncoMouseTm: Feminism and Technoscience. Nueva York, Routledge, 1997.

HARDING, Sandra. Ciencia y feminismo. Barcelona, Morata, 1996 (1986).

HARDING, Sandra. Whose Science? Whose Knowledge? Ithaca, NY, Cornell University Press, 1991.

HEINZ, Bettina et alii. Under the Rainbow Flag: Webbing Global Gay Identities. International Journal of Sexuality and Gender Studies, $\mathrm{n}^{\circ} .7$, 2002, pp.107-24. DOI:10.1023/a:1015841032097.

HIMANEN, Pekka. La ética hacker y el espíritu de la era de la información. Barcelona, Destino, 2004 (2001).

KVASNY, Lynette. Let the Sisters Speak: Understanding Information Technology from the Standpoint of the 'Other'. The DATA BASE for Advances in Information Systems, vol.37, n'.4, 2006, pp.13-25. DOI: $\underline{10.1145 / 1185335.1185342 .}$

KELLER, Eveling. Reflexiones sobre género y ciencia, Valencia, Alfons el Magnànim, 1991 (1985).

KINKI, Klau. Ofensiva transhackfeminista. Your machine is a battlerground: En: SOLÁ, Miriam; URKO, Elena (Comp.). 
Transfeminismos. Epistemes, fricciones y flujos. Tafalla Nafarroa, Txalaparta, 2013, pp.313-325.

LANDSTRÖM, Catharina. Queering feminist technology studies. Feminist Theory, vol.8, $\mathrm{n}^{\circ} .1, \quad 2007, \quad$ pp.7-26. DOI: $10.1177 / 1464700107074193$.

LEGGon, Cheryl B. Gender, Race/Ethnicity, and the Digital Divide. In: Fox, Mary F; Johnson, DeboraH G; Rosser, Sue (eds.). Women, Gender, and Technology, Urbana, University of Illinois, 2006.

LESSIG, Lawrence. Code and Other Laws of Cyberspace. New York, Basic Books, 1999.

MARCHANTE, Diego. Transbutch. Luchas fronterizas de género entre el arte y la política. Tesis doctoral. Barcelona, Universitat Autònoma de Barcelona, 2015. [http://diposit.ub.edu/dspace/bitstream/2445/97243/1/01.AMH_1de2. pdf -consultado 15 agos. 2018].

Merchant, Carolyn. The Death of Nature. Women, Ecology, and the Scientific Revolution. New York, Harper Collins, 1980.

MIES, María; SHIVA, Vandana. Ecofeminism. Canadá, Femwood, 1993.

NAKAMURA, Lisa. Cybertypes: Race, Ethnicity, and Identity on the Internet. New York, Routledge, 2002.

NIP, Joyce Y. M. The Relationship Between Online and Offline Communities: The Case of the Queer Sisters. Media, Culture, and Society, vol.26, $\mathrm{n}^{\circ} .3, \quad 2004, \quad$ pp.409-28. DOI: 10.1177/0163443704042262.

OAKLEY, Ann. The Sociology of Housework, Londres, Martin Robertson, 1974.

O'RIORDAN, Kate; PHILLIPS, David J. Queer online: Media Technology and Sexuality. New York, Peter Lang Publishers, 2007.

ORTMAN, Cecilia. Software Libre, géneros y (des)igualdad: expandir los horizontes de la libertad. Mujeres en red. El periódico feminista, 2016. [http://www.mujeresenred.net/spip.php?article2267 -consultado en: 27 jul. 2018].

Plant, Sadie. Zerost ones: digital woment the new technoculture. London, Fourth Estate, 1997. 
PERDOMO, Inmaculada. Género y tecnologías. Ciberfeminismo y construcción de la tecnocultura actual. Revista Iberoamericana de Ciencia, Tecnología y Sociedad, vol.11, n.31, 2016, pp.171-193. [http://www.scielo.org.ar/scielo.php?script=sci abstract\&pid $=\mathrm{S} 18500$ 0132016000100009 -consultado en: 12 may. 2018].

PHILIP, Kavita. ¿Tecnologías para pobres o tecnologías pobres? Poscolonialismo, desarrollo y tecnología en India. Nomadas, $\mathrm{n}^{\circ} .36$, 2012 , pp.91-108. [http://www.redalyc.org/articulo.oa?id=105124264007 -consultado en: 13 jun. 2018].

PRECIADO, Paul. Testo Yonqui. Madrid, Espasa, 2008.

POST-OP. De placeres y monstruos. Interrogantes en torno al postporno. En: SOLÁ, Miriam; URKO Elena (coords.). Transfeminismos. Epistemes, fricciones y flujos. Tafalla Nafarroa, Txalaparta, 2013, pp.193-210.

PUJOL, Joan; MonTENEGRO, Marisela. Technology and Feminism: a Stranger Couple. Revista de Estudios Sociales, no 51, 2015, pp.173185. DOI:10.7440/res51.2015.14.

ROSE, Hilary. Love, Power and Knowledge: Towards a Feminist Transformation of the Sciences. Bloomington, IN, Indiana University Press, 1994.

SCHWARTZ, Ruth. More Work for Mother: The Ironies of Household Technology from the Open Hearth to the Microwave. New York, Basic Books, 1983.

SCHIEBINGER, Londa. Nature's Body. Gender in the Making of Modern Science, Boston, Beacon Press, 1993.

SORIA, Irene. El software libre y la cultura hacker como vehículo para la emancipación tecnológica y su vínculo con la lucha feminista. En: SORIA, Irene (Coord.). Ética Hacker, seguridad y vigilancia. México D.F, Universidad del Claustro de Sor Juana, 2016, pp.183-216.

STONE, Allucquère Rosanne "Sandy". Will the Real Body Please Stand Up?: Boundary Stories about Virtual Cultures. In: BENEDIKT, Michael (ed.). Cyberspace: First Step. Cambridge, MIT Press, 1991, pp.81118. 
STONE, Allucquère Rosanne "Sandy". The War of Desire and Technology at the Close of the Mechanical Age. Cambridge, MIT Press, 1995.

TAPIA, Danae. Por un ciberfeminismo interseccional en América Latina. Revista Pillku, vol.18, 2015. [https://pillku.org/article/por-unciberfeminismo-interseccional-en-america-la/ - consultado en: 14 jul. 2018].

TEJIDOS Hackfeministas. El desafío de una tecnología feminista: la reconfiguración necesaria. Surgiendo. Comunicación y cultura digital, $2014 . \quad$ [https://sursiendo.com/blog/2014/04/el-desafio-de-unatecnologia-feminista-la reconfiguracion-necesaria/ - consultado en: 15, agos. 2018].

THOMAS, Douglas. Hacker Culture. Minneapolis, University of Minnesota Press,2002.

THOMAS, Douglas. Hacking the Body: Code, Performance and Corporeality. New Media Society, vol. 7, n.5, 2005, pp.647662.DOI: $10.1177 / 1461444805056010$.

TOUPIN, Sophie. Feminist Hackerspaces. The Synthesis of Feminist and Hacker Culture. Journal of Peer Production, 2014 [https://www.academia.edu/24232869/Feminist Hackerspaces The S ynthesis of Feminist and Hacker_Cultures 2014 _ consultado en: 29 ago. 2018]

VERGÉS, Nuria. Teorías principales de las tecnologías: Evolución y principales debates. Barcelona, Universidad de Barcelona, 2013. [http://diposit.ub.edu/dspace/handle/2445/45624 - consultado en: 24 may. 2018]

Vergés, Núria; HACHE, Alex; Cruells, Eva. Ciberfeminismo de Investigacción. El caso de donestech. En: ZAFRA; Remedios (coord.). xOy1\# Ensayos sobre género y ciberespacio. Madrid: Briseño Editores, 2010, pp.173-189.

Vergés, Núria; HACHE, Alex; CRUElls, Eva. Ciberfeminismo de investigacción con y entre tecnoartistas y hackers. Athenea Digital. Revista de pensamiento e Investigación Social, vol. 14, n. 4, 2014, pp. 153-180. DOI:10.5565/rev/athenea.1352.

WAJCMAN, Judith. Feminist confronts technology. USA, Pennsylvania State University Press, 1991. 
WAJCMAn, Judith. Technofeminism. Oxford, Polity Press, 2004.

WAJCMAN, Judith. From Women and Technology to Gendered Technoscience. Information, Communication \& Society, vol.10, n.3, 2007, pp.287-298. DOI:10.1080/13691180701409770 .

WAJCMAN, Judith. Feminist theories of technology. Cambridge Journal of Economics, vol.34, n.1, 2009, pp.143-152. DOI:10.1093/cje/ben057

WAKEFORD, Nina. Cyberqueer. In: BELL, David; KeNNEDY, Barbara (eds.). The Cybercultures Reader. London and New York, Routledge, 2000, pp.403-415.

WAKEFORD, Nina. New technologies and cyber-queer research. In RICHARSON, Diane; SEIDMAN, Steven (eds). Handbook of lesbian and gay studies. London, Sage Publications, 2002, pp.115-144.

Wright, Michelle M. Racism, Technology, and the Limits of Western Knowledge. In: FERNÁNDEZ, Maria; WILDING, Faith; WRIGHT, Michelle M (eds.). Domain Errors! Cyberfeminist practices. Brooklyn, N.Y, Autonomedia, 2002, pp.45-61.

WRIGHT, Michelle M. Finding a Place in Cyberspace: Black Women, Technology, and Identity. Frontiers, vol.26, n'.1, 2005, pp.48-59. DOI:10.1353/fro.2005.0017.

Woodland, Randal. Queer Spaces, Modem Boys and Pagan Statues: Gay/Lesbian Identity and the Construction of Cyberspace. In: BELL, David; KENNEDY, Barbara M (eds.). The Cybercultures Reader. London, Routledge, 2000, pp.416-431.

Wolmark, Jenny (ed.). Cybersexualities: A Reader on Feminist Theory, Cyborgs, and Cyberspace. Edinburgh, Edinburgh University Press, 1999.

ZAFRA, Remedios. Un cuarto propio conectado. Feminismos y creación desde la esfera público-privada on-line. Asparkía Investigació Feminista, $\quad \mathrm{n}^{\circ} \quad 22, \quad 2011$, pp.115-129. [http://www.erevistes.uji.es/index.php/asparkia/article/view/602/513 consultado en: 4 may. 2018]. 